\title{
Receipt of tobacco direct mail/email discount coupons and trajectories of cigarette smoking behaviours in a nationally representative longitudinal cohort of US adults
}

\author{
Kelvin Choi, ${ }^{\oplus 1}$ Julia Cen Chen, ${ }^{1}$ Andy S L Tan, ${ }^{\circledR 2,3}$ Samir Soneji, ${ }^{4}$ Meghan B Moran ${ }^{5}$
}

\begin{abstract}
${ }^{1}$ Division of Intramural Research, National Institute on Minority Health and Health Disparities, Bethesda, Maryland, USA ${ }^{2}$ Division of Population Sciences, Dana-Farber Cancer Institute, Boston, Massachusetts, USA ${ }^{3}$ Department of Social and Behavioral Sciences, Harvard T.H. Chan School of Public Health, Boston, Massachusetts, USA ${ }^{4}$ Geisel School of Medicine at Dartmouth, The Dartmouth Institute for Health Policy \& Clinical Practice, Lebanon, New Hampshire, USA

${ }^{5}$ Department of Health, Behavior and Society, Johns Hopkins University Bloomberg School of Public Health, Baltimore, Maryland, USA
\end{abstract}

\section{Correspondence to} Dr Kelvin Choi, Division of Intramural Research, National Institute on Minority Health and Health Disparities, Bethesda, MD 20892, USA;

kelvin.choi@nih.gov

Received 9 March 2018 Revised 10 May 2018

Accepted 11 May 2018

Published Online First

19 June 2018

\section{ABSTRACT}

Background We assessed whether receipt of coupons — via direct mail or e-mail—was prospectively related to trajectories of smoking behaviours.

Methods Data were from a cohort of US adults ( $n=26447)$ who participated in wave 1 (2013-2014) and wave 2 (2014-2015) of the Population Assessment of Tobacco and Health Study. Participants reported receipt of tobacco direct mail/email coupons in the past 6 months in wave 1 and their smoking status in both waves. Weighted multiple logistic regressions were used to examine demographic correlates of receiving tobacco direct mail/email coupons at wave 1 and to examine the prospective effect of receiving tobacco coupons on trajectories of smoking behaviours.

Findings At wave 1, 10.7\% of never smokers, 13.9\% of experimental smokers, $37.1 \%$ of current smokers and $16.5 \%$ of former smokers reported receiving tobacco direct mail/email coupons. Lower education and higher poverty adults and non-Hispanic white current smokers were more likely to have received these coupons $(p<0.05)$. Receiving tobacco direct mail/email coupons at wave 1 was associated with increased odds of smoking initiation among never smokers (adjusted odds ratio $(A O R)=2.28,95 \% \mathrm{Cl} 1.36$ to 3.83 ), becoming established smokers among experimenters ( $A O R=1.62$, $95 \% \mathrm{Cl} 1.29$ to 2.04), becoming daily smokers among non-daily smokers ( $A O R=1.56,95 \% \mathrm{Cl} 1.23$ to 1.99) and smoking relapse among former smokers between waves (AOR=1.91, 95\% Cl 1.39 to 2.65). Receiving these coupons at wave 1 was associated with reduced odds of smoking cessation $\geq 6$ months among current smokers ( $A O R=0.71,95 \% \mathrm{Cl} 0.58$ to 0.88 ).

Conclusions Tobacco direct mail/email coupons encourage and sustain smoking and disproportionately affect lower socioeconomic populations.

\section{INTRODUCTION}

Tobacco use, primarily cigarette smoking, was responsible for 100 million deaths globally in the 20th century and is expected to claim an additional one billion lives worldwide in the 21 st century. ${ }^{1}$ Tobacco marketing has been found to be causally related to smoking behaviours. ${ }^{23}$ One little examined marketing strategy, however, is the dissemination of promotional discounts, such as coupons, to consumers (figure 1). While the US tobacco industry spends a relatively small proportion of its marketing budget on this strategy (US\$346 million in 2014, 3.9\% of the total marketing expenditure), ${ }^{45}$ previous studies have found that receiving these discount coupons was associated with smoking behaviours in adults. For example, in a prospective cohort study among Minnesota young adults, exposure to tobacco direct mail marketing $(86.5 \%$ of these materials contained at least one discount coupon ${ }^{6}$ ) was associated with higher odds of non-smokers becoming current smokers and lower odds of current smokers quitting smoking. ${ }^{7}$ A recent study among a US nationally representative sample of adults similarly reported associations between exposure to tobacco discount coupons and increased smoking behaviours. ${ }^{8}$ Other studies also found that exposure to tobacco direct mail/email coupons is associated with susceptibility to using snus ${ }^{9}$ and electronic cigarettes. ${ }^{10}$ Globally, although the WHO Framework Convention on Tobacco Control (FCTC) recommends bans on tobacco marketing, with an emphasis on banning promotional discounts, 84 countries (with $49 \%$ of the world's population) implemented a ban on promotional discounts. ${ }^{11}$ The USA does not have a ban on tobacco promotional discounts. ${ }^{11}$ While prohibiting distribution of these coupons in the USA would be difficult because of protection of commercial speech under the First Amendment and protection of interstate commerce by the Commerce Clause, prohibiting redemption of these coupons is feasible and has withstood legal challenges in the USA. ${ }^{12}$

Furthermore, the tobacco industry's distribution of tobacco direct mail/email coupons disproportionately impacts vulnerable populations within the USA. For instance, previous US national and regional studies have found that adults from lower socioeconomic status (SES) populations (defined either by education, income or poverty status) were more likely to receive tobacco direct mail/email coupons. ${ }^{7-9}$ As revealed in analyses of the tobacco industry's internal documents, ${ }^{13}$ the tobacco industry deliberately targets lower SES populations with tobacco discount coupons. Previous studies showed that $35.8 \%$ of Minnesota young adults and $80.1 \%$ of Minnesota adult smokers who receive tobacco direct mail/email coupons redeem these coupons for tobacco products. ${ }^{14}$ Thus, the disproportionate receipt of these coupons among lower SES individuals could perpetuate tobacco-related health disparities among socioeconomically vulnerable populations.

However, the current literature is limited in the following ways. First, there is a lack of nationally representative longitudinal evidence regarding the effect of tobacco coupons on tobacco use trajectories, with only one retrospective study examining 


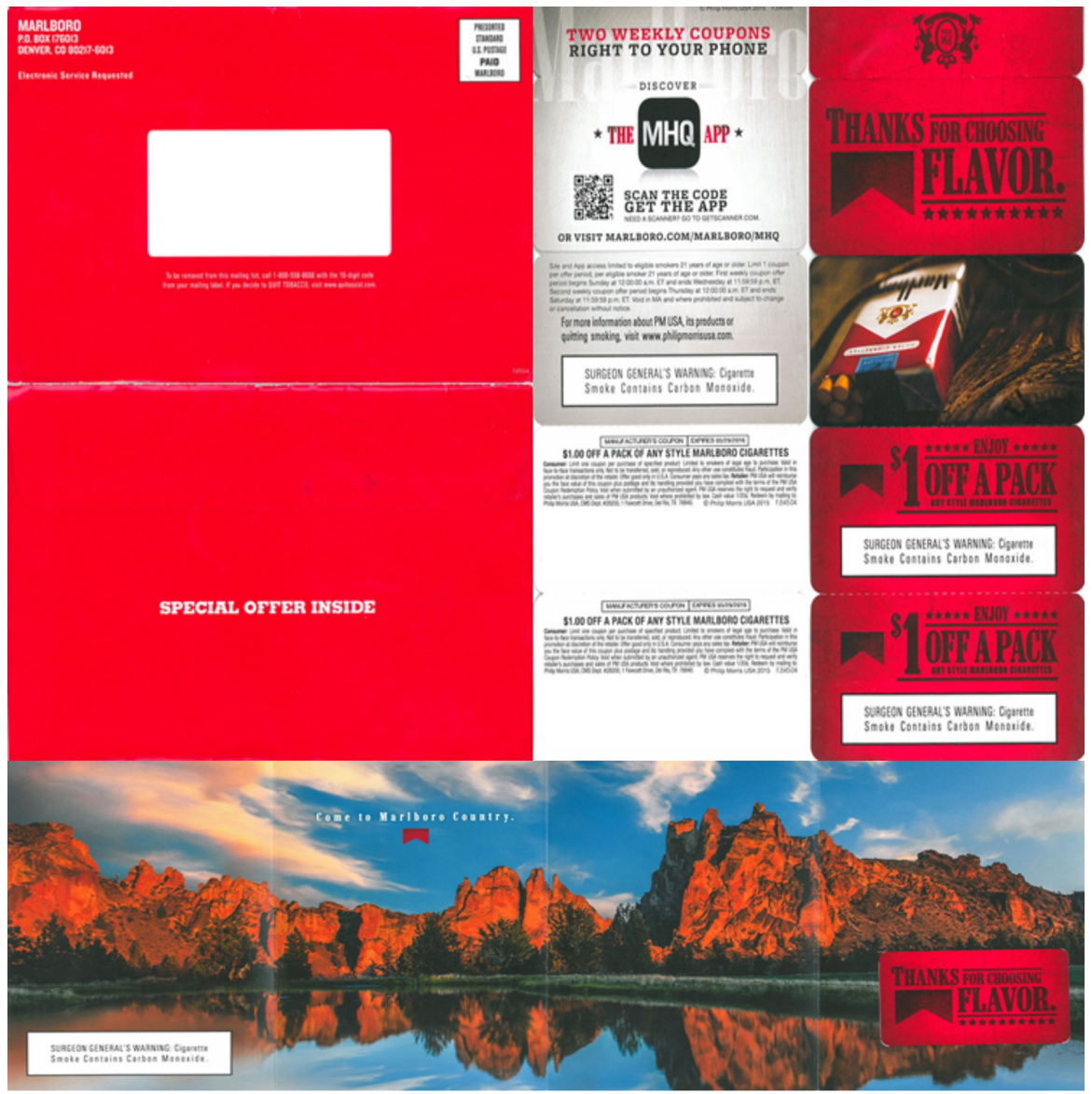

Figure 1 An example of tobacco direct mail coupons. Source: www.trinketsandtrash.org.

this relationship using cross-sectional data from a US nationally representative adult sample. ${ }^{8}$ Second, this previous national study did not examine how receipt of these coupons influences individuals at different stages of smoking behaviour development (eg, never smoking, trying, experimentation, established use and daily use) nor the process of smoking cessation or relapsing. Third, most of the studies that examined the effects of tobacco marketing on smoking progression have focused on the youth population, ${ }^{15}$ and far less has been done to investigate how marketing influences smoking trajectory among adults. To overcome these limitations, the current study analysed longitudinal data collected through the Population Assessment of Tobacco and Health (PATH) Study to examine the potential disparities in receipt of tobacco direct mail/email coupons among vulnerable populations, and how receipt of tobacco direct mail/email coupons influence trajectories of smoking behaviours among US adults. Findings may help inform potential regulation of this type of tobacco marketing.

\section{METHODS}

\section{Study design and participants}

Data used in this study were from waves 1 and 2 of the PATH Study, adult sample conducted from September 2013 to December 2014 and October 2014 to October 2015, respectively. The PATH Study is a nationally representative, longitudinal cohort study of tobacco use among youth and adults in the USA. ${ }^{16}$ The study used a four-stage stratified area probability sample design, with a two-phase design for sampling adults at the final stage. ${ }^{16}$ The study sampled over 150000 mailing addresses, which yielded a sample of 32320 adult respondents who completed the wave 1 interview. ${ }^{17}$ Adult respondents completed the wave 2 interview approximately 1 year after the wave 1 interview, and the unweighted and weighted retention rates between the two waves were $82.6 \%$ and $83.1 \%$, respectively. ${ }^{17}$ Consequently, a sample of 26447 adults completed both waves 1 and 2 interviews, and their responses were analysed in this study. Interviews were administered using audio computer-assisted self-interviewing.

\section{Measures}

Smoking status and trajectories

To investigate development of smoking behaviours, never smokers were participants who responded 'No' to the question 'Have you ever smoked a cigarette, even one or two puffs?' at wave 1 . Among these never smokers, those who reported that they ever smoked a cigarette at wave 2 were classified as initiated smoking. Experimenters were participants who responded 'Yes' to have ever smoked a cigarette and reported having smoked less than 100 cigarettes in their lifetime at wave 1. Among these experimenters, those who reported that they smoked 100 cigarettes or more in their lifetime at wave 2 were classified as becoming established smokers. Non-daily smokers were participants who reported having smoked 100 cigarettes or more in their lifetime and responded 'Some days' to the question 'Do you now smoke cigarettes?' at wave 1. Among these non-daily smokers, those who reported 'Every day' to this question at wave 2 were classified as becoming daily smokers. Current smokers were participants who reported having smoked 100 cigarettes or more in their lifetime and currently smoking every day or some days at wave 1. Among these current smokers, those who 
reported not currently smoking and reported 'More than past 6 months' to the question 'About how long has it been since you completely quit smoking cigarettes?' at wave 2 were classified as having quit smoking. Lastly, participants who had smoked 100 cigarettes or more in their lifetime and did not currently smoke at all at wave 1 were classified as former smokers. Among these former smokers, those who reported currently smoking every day or some days at wave 2 were classified as having relapsed.

\section{Receipt of tobacco direct mail/email coupons at wave 1}

In the wave 1 interview, participants were asked, 'In the past 6 months, have you received an e-mail message with promotions or coupons for cigarettes or tobacco products?' and 'In the past 6 months, have you received promotions or coupons for cigarettes or tobacco products in the mail?'. Participants who responded 'Yes' to either of these questions were classified as having received direct mail/email coupons; participants who answered 'No' to both questions were classified as not having received direct mail/email coupons.

\section{Sociodemographic characteristics at wave 1}

We included age, gender, race/ethnicity (non-Hispanic white, non-Hispanic black, Hispanic and non-Hispanic other), education level (<high school or general equivalency diploma (GED) holder, high school graduate, some college with no degree and bachelor's degree or above), poverty level (<100\%, 100\%$199 \%$ and $\geq 200 \%$ of poverty line) and census region (northeast, south, midwest and west) as covariates in the analyses.

\section{Statistical analysis}

All analyses were stratified by smoking status at wave 1 (never smokers, experimenters, non-daily smokers, current smokers and former smokers). We first examined the correlates of receiving direct mail/email coupons for each smoking status using multiple logistic regressions models including all sociodemographic variables. To investigate the associations between receiving direct mail/email coupons and trajectories of smoking, we used multiple logistic regression models, adjusting for all sociodemographic variables. We fitted five separate logistic regression models, adjusting for sociodemographic variables to analyse whether receiving coupons at wave 1 predicted changes in smoking statuses between waves: (1) initiating smoking among never smokers, (2) becoming established smokers among experimenters, (3) becoming daily smokers among non-daily smokers, (4) quit smoking among current smokers and (5) relapse among former smokers. In the smoking cessation analysis, we performed a sensitivity analysis by including time-to-first-cigarette assessed in wave 1 as a nicotine dependence measure in the model. Since the results did not change significantly, we chose not to include nicotine addiction measures for model parsimony. We used the Stata V.14.0 survey command to employ the wave 2 balanced repeated replications weights with Fay's adjustment $(p=0.3)$, accounted for the non-response from wave 1 to wave $2 .{ }^{17}$

\section{RESULTS}

Table 1 presents the demographics of the sample by smoking status at wave 1 . The overall sample was $52.1 \%$ female, $65.7 \%$ non-Hispanic white, $31.0 \%$ with some college education but no degree, $46.8 \%$ at or above $200 \%$ of the poverty line and $37.2 \%$ from the US south region. Overall, $17.5 \%$ of the sample, $10.7 \%$ of never smokers, $13.9 \%$ of experimental smokers, $26.6 \%$ of non-daily smokers, $37.1 \%$ of current smokers and $16.5 \%$ of former smokers reported receiving tobacco direct mail/email coupons in the past 6 months.

Table 2 presents the association between demographics and receipt of tobacco direct mail/email coupons by smoking status at wave 1. Individuals with some college education, regardless of smoking status, were more likely than those with a bachelor's degree to have received tobacco direct mail/email coupons $(\mathrm{p}<0.05)$. Similarly, individuals less than the $200 \%$ poverty line, regardless of smoking status, were more likely than those at or above the $200 \%$ poverty line to have received tobacco direct mail/email coupons, except among non-daily smokers $(\mathrm{p}<0.05)$. Some minority populations were less likely than non-Hispanic white participants to have received these coupons, for example, among never smokers and current smokers $(\mathrm{p}<0.05)$. However, non-Hispanic black experimenters were more likely than non-Hispanic white experimenters to have received tobacco direct mail/email coupons $(p<0.05)$. Male current smokers were less likely than female current smokers to have received tobacco direct mail/email coupons $(p<0.05)$. Compared with their counterparts living in the West, experimenters and non-daily smokers living in the Midwest and the South and current smokers living in the Midwest were more likely to have received tobacco direct mail/email coupons $(\mathrm{p}<0.05)$.

Table 3 presents the weighted prevalence of smoking trajectories at wave 2 by wave 1 smoking status and receipt of tobacco direct mail/email coupons, and figure 2 summarises the associations between exposure to tobacco direct mail/email coupons and smoking behaviours. For example, among wave 1 never smokers, $4.0 \%$ of those who reported receiving tobacco direct mail/email coupons initiated smoking by wave 2 , while $2.0 \%$ of those who reported not receiving tobacco direct mail/email coupons initiated smoking by wave $2(\mathrm{AOR}=2.28,95 \%$ CI 1.36 to 3.83 ; figure 2A). Furthermore, having received tobacco direct mail/ email coupons was associated with increased odds of becoming established smokers among wave 1 experimenters $(\mathrm{AOR}=1.62$, $95 \%$ CI 1.29 to 2.04; figure $2 \mathrm{~B}$ ), and becoming daily smokers among wave 1 non-daily smokers (AOR $=1.56,95 \%$ CI 1.23 to 1.99; figure $2 \mathrm{C}$ ), and relapse among wave 1 former smokers $(\mathrm{AOR}=1.91,95 \%$ CI 1.39 to 2.65 ; figure $2 \mathrm{E})$. Additionally, having received tobacco direct mail/email coupons was associated with reduced odds of smoking cessation among wave 1 current smokers (AOR $=0.71,95 \% \mathrm{CI} 0.58$ to 0.88 ; figure $2 \mathrm{D}$ ).

\section{DISCUSSION}

This is the first US national study that details the influence of tobacco direct mail/email coupons on smoking initiation, progression, cessation and relapses. Our findings, together with previous studies, ${ }^{7814}$ further support an urgent need for prohibiting tobacco direct mail/email coupons, especially when further analysis of the PATH Study data showed that only 5.1\% of adult non-smokers and $9.9 \%$ of adult current smokers at wave 1 reported signing up for email alerts and other promotions for tobacco products. This regulatory action is aligned with the WHO FCTC, the first international treaty to combat this global tobacco use epidemic using evidence-based strategies. For example, the Monitor tobacco use, Protect people from tobacco use, Offer help to quit tobacco use, Warn about the dangers of tobacco, Enforce ban on tobacco marketing, and Raise tobacco taxes (MPOWER) model, introduced by the WHO in $2008,{ }^{18}$ highlights banning promotional discounts as one important component of enforcing tobacco marketing restrictions. At present, about half of the countries that ratified the WHO FCTC did not ban tobacco price promotion strategies 


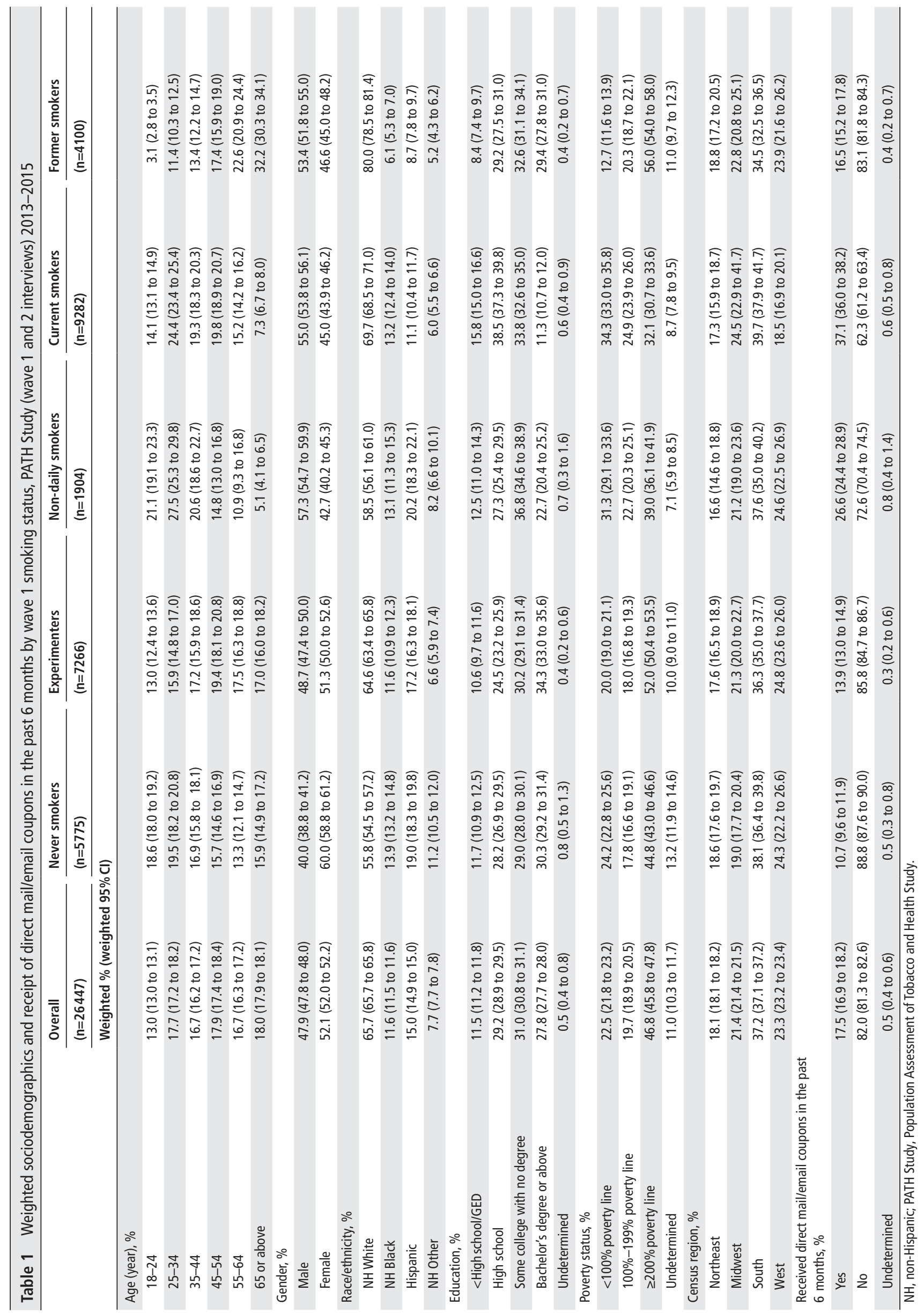


Table 2 Weighted associations between sociodemographics and receipt of direct mail/email coupons in the past 6 months by wave 1 smoking status, PATH Study (wave 1 and 2 interviews) 2013-2015

\begin{tabular}{|c|c|c|c|c|c|}
\hline & \multicolumn{5}{|c|}{ Receipt of direct mail/email coupons in the past 6 months* } \\
\hline & Never smokers & Experimenters & Non-daily smokers & Current smokers & Former smokers \\
\hline & \multicolumn{5}{|c|}{ Weighted AOR (weighted $95 \% \mathrm{Cl}$ ) } \\
\hline \multicolumn{6}{|l|}{ Age (year) } \\
\hline $18-24$ & 1.14 (0.77 to 1.69$)$ & $1.31(0.94$ to 1.83$)$ & 1.51 (0.78 to 2.90$)$ & $1.53(1.19$ to 1.96$)$ & 2.29 (1.55 to 3.39$)$ \\
\hline $25-34$ & 1.87 (1.24 to 2.82$)$ & $2.08(1.46$ to 2.96$)$ & 2.65 (1.41 to 4.99$)$ & $2.98(2.34$ to 3.80$)$ & $2.94(2.12$ to 4.07$)$ \\
\hline $35-44$ & $1.76(1.09$ to 2.84$)$ & $1.93(1.26$ to 2.98$)$ & 2.09 (1.04 to 4.12$)$ & $2.79(2.15$ to 3.61$)$ & 2.64 (1.79 to 3.88$)$ \\
\hline $45-54$ & 2.07 (1.32 to 3.25$)$ & $2.42(1.73$ to 3.40$)$ & 2.29 (1.18 to 4.43$)$ & 2.41 (1.81 to 3.20$)$ & 2.50 (1.79 to 3.50$)$ \\
\hline $55-64$ & 1.64 (1.03 to 2.62$)$ & 1.64 (1.09 to 2.48$)$ & 1.99 (1.09 to 3.64$)$ & 1.74 (1.32 to 2.29$)$ & 1.65 (1.15 to 2.37$)$ \\
\hline $65-74$ & Reference & Reference & Reference & Reference & Reference \\
\hline \multicolumn{6}{|l|}{ Gender } \\
\hline Male & $0.88(0.73$ to 1.08$)$ & $1.02(0.86$ to 1.21$)$ & $0.78(0.62$ to 0.98$)$ & $0.70(0.63$ to 0.77$)$ & $0.87(0.69$ to 1.10$)$ \\
\hline Female & Reference & Reference & Reference & Reference & Reference \\
\hline \multicolumn{6}{|l|}{ Race/ethnicity } \\
\hline NH white & Reference & Reference & Reference & Reference & Reference \\
\hline NH black & 1.28 (0.99 to 1.65$)$ & $1.29(1.04$ to 1.59$)$ & 0.90 (0.62 to 1.29$)$ & $0.80(0.67$ to 0.94$)$ & $0.87(0.61$ to 1.25$)$ \\
\hline Hispanic & $0.60(0.40$ to 0.91$)$ & 0.79 (0.61 to 1.04$)$ & 0.69 (0.51 to 0.93$)$ & $0.60(0.49$ to 0.68$)$ & 0.79 (0.58 to 1.09$)$ \\
\hline NH others & $0.53(0.29$ to 0.96$)$ & $0.96(0.67$ to 1.41$)$ & $0.86(0.54$ to 1.37$)$ & $0.72(0.57$ to 0.90$)$ & $0.74(0.47$ to 1.19$)$ \\
\hline \multicolumn{6}{|l|}{ Education } \\
\hline$<$ High school/GED & $1.34(0.85$ to 2.13$)$ & $1.10(0.76$ to 1.57$)$ & $1.16(0.73$ to 1.85$)$ & 1.07 (0.87 to 1.31$)$ & $0.87(0.50$ to 1.43$)$ \\
\hline High school & 1.32 (0.97 to 1.81$)$ & 1.49 (1.13 to 1.96$)$ & 1.34 (0.94 to 1.93$)$ & 1.26 (1.04 to 1.52$)$ & 1.65 (1.26 to 2.15$)$ \\
\hline Some college with no degree & 1.76 (1.32 to 2.35$)$ & $1.49(1.17$ to 1.89$)$ & 1.48 (1.09 to 2.02 ) & 1.29 (1.08 to 1.55$)$ & $1.49(1.13$ to 1.97$)$ \\
\hline Bachelor's degree or above & Reference & Reference & Reference & Reference & Reference \\
\hline Undetermined & $1.45(0.19$ to 11.13$)$ & $0.74(0.11$ to 5.01$)$ & 0.16 (0.01 to 2.67$)$ & 1.02 (0.34 to 3.07$)$ & $-\dagger$ \\
\hline \multicolumn{6}{|l|}{ Poverty tatus } \\
\hline$<100 \%$ poverty line & $1.17(0.84$ to 1.63$)$ & $1.73(1.37$ to 2.20$)$ & $1.09(0.81$ to 1.45$)$ & $1.18(1.04$ to 1.33$)$ & 1.45 (1.06 to 1.98$)$ \\
\hline $100 \%-199 \%$ poverty line & $1.47(1.14$ to 1.90$)$ & $1.42(1.10$ to 1.84$)$ & $1.28(0.90$ to 1.82$)$ & 1.26 (1.08 to 1.46$)$ & 1.19 (0.89 to 1.58$)$ \\
\hline$\geq 200 \%$ poverty line & Reference & Reference & Reference & Reference & Reference \\
\hline Undetermined & $0.77(0.48$ to 1.24$)$ & $0.57(0.34$ to 0.95$)$ & $0.84(0.50$ to 1.41$)$ & $0.73(0.60$ to 0.89$)$ & $0.64(0.42$ to 0.99$)$ \\
\hline \multicolumn{6}{|l|}{ Census region } \\
\hline Northeast & $0.73(0.49$ to 1.08$)$ & 1.18 (0.86 to 1.62$)$ & $0.86(0.52$ to 1.41$)$ & $0.96(0.82$ to 1.11$)$ & $0.96(0.74$ to 1.27$)$ \\
\hline Midwest & 0.99 (0.66 to 1.48$)$ & $1.36(1.04$ to 1.77$)$ & 1.58 (1.16 to 2.16 ) & 1.24 (1.07 to 1.44$)$ & $1.21(0.91$ to 1.61$)$ \\
\hline South & 0.85 (0.60 to 1.21$)$ & $1.48(1.21$ to 1.80$)$ & 1.57 (1.14 to 2.15$)$ & 1.08 (0.95 to 1.24$)$ & 1.07 (0.79 to 1.44$)$ \\
\hline West & Reference & Reference & Reference & Reference & Reference \\
\hline
\end{tabular}

Note: adjusted for all socioeconomic variables in the table. Bold estimates are statistically significant $(p<0.05)$.

${ }^{*}$ The undetermined category of receiving direct mail/email coupons was not included in the regression models.

tThe undetermined category for education was omitted because of small sample size and wide $\mathrm{Cl}$.

AOR, adjusted OR; NH, non-Hispanic; PATH Study, Population Assessment of Tobacco and Health Study.

in their country, ${ }^{18}$ leaving their populations vulnerable to this marketing strategy.

There is evidence to suggest that banning tobacco coupons and other types of price promotions can be effective in eliminating the impact of tobacco direct mail/email coupons on smoking behaviours. For example, a previous study compared the association between repeated exposure to tobacco price promotions and current smoking among baseline current and former smokers between countries with and without a ban on tobacco price promotions. ${ }^{19}$ This study found that among countries without such a ban, exposure to tobacco price promotions was positively associated with continuation of smoking. However, this association was not observed among countries with a tobacco price promotion ban. ${ }^{19}$ It is noteworthy that prohibiting distribution of tobacco discount coupons will be difficult. Under the First Amendment, the tobacco companies have protection rights to commercial speech,

Table 3 Weighted prevalence of smoking trajectories at wave 2 by wave 1 smoking status and receipt of tobacco direct mail/email coupons, PATH Study (wave 1 and 2 interviews) 2013-2015

\begin{tabular}{llllll}
\hline & Never smokers & Experimenters & Non-daily smokers & Current smokers & Former smokers \\
\cline { 2 - 6 } & Weighted prevalence \% (weighted $95 \% \mathrm{Cl})$ & & & \% Relapsed \\
\hline $\begin{array}{l}\text { Received direct mail/email } \\
\text { coupons in the past } 6 \text { months }\end{array}$ & $\begin{array}{l}\text { \% Initiated smoking } \\
\text { Yes }\end{array}$ & $\begin{array}{l}\text { \% Became established } \\
\text { smokers }\end{array}$ & \% Became daily smokers & \% Quit smoking & \\
No & $4.0(2.7$ to 6.0$)$ & $17.3(14.8$ to 20.1$)$ & $30.7(26.9$ to 34.9$)$ & $4.2(3.5$ to 5.2$)$ & $11.5(9.1$ to 14.7$)$ \\
\hline
\end{tabular}




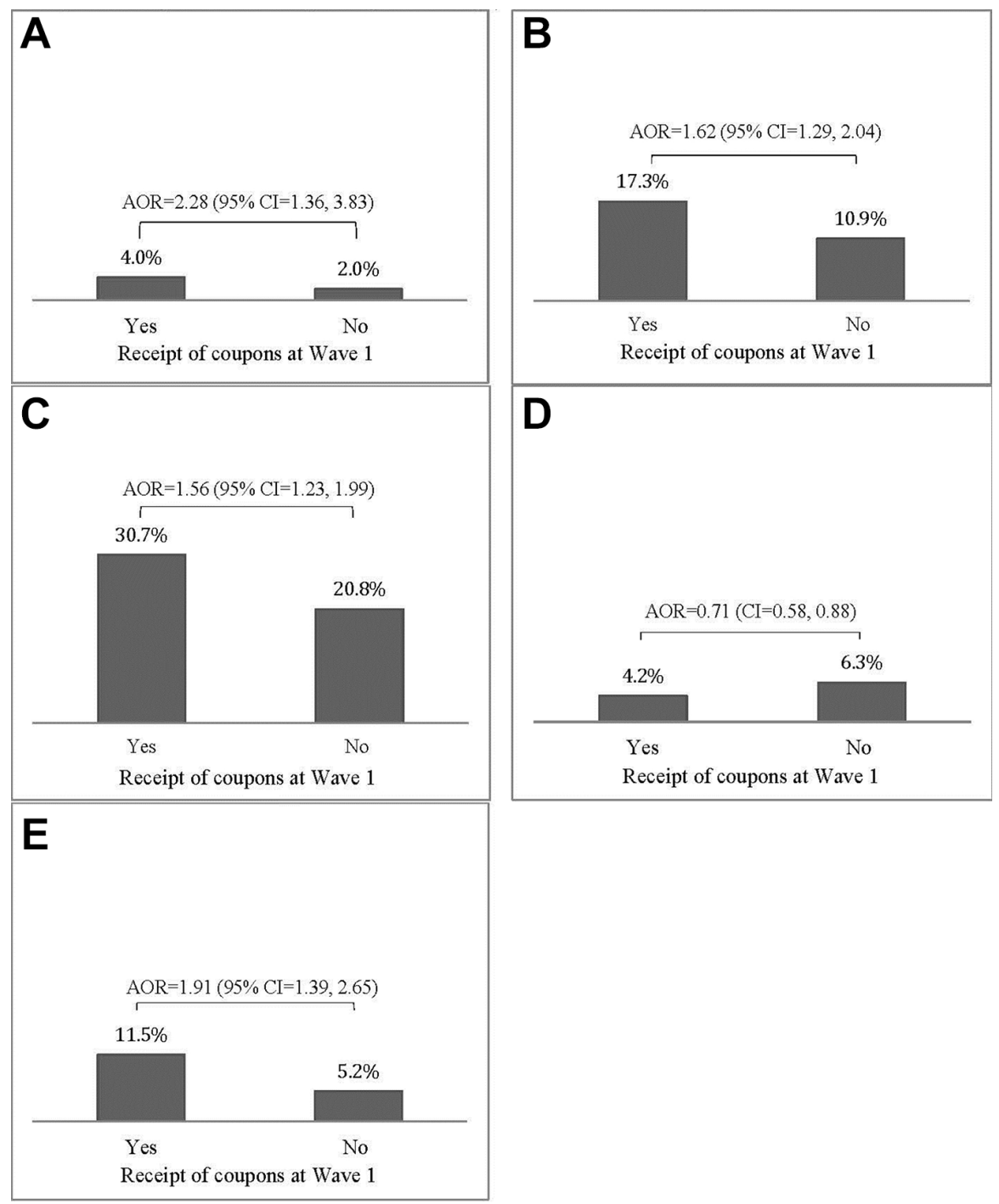

Figure 2 Weighted adjusted associations between receipt of tobacco direct mail/email coupons in the past 6 months and wave 2 smoking behaviour by wave 1 smoking status, PATH Study (wave 1 and 2 interviews) 2013-2015. (A) Proportion of wave 1 never smokers initiated smoking by wave 2. (B) Proportion of wave 1 experimenters became established smokers by wave 2. (C) Proportion of wave 1 non-daily smokers became daily smokers by wave 2. (D) Proportion of wave 1 current smokers quit smoking by wave 2. (E) Proportion of wave 1 former smoker relapsed to smoking by wave 2 . AOR, adjusted $O R$.

and communicating price information with consumers could fall under this protection. Additionally, under the Commerce Clause, interstate commerce is protected, and prohibiting distribution of these coupons would be challenging if they are distributed across state lines. In contrast, prohibiting redemption of tobacco discount coupons would be feasible. ${ }^{12}$ For example, New York City implemented a policy in 2014 to prohibit redemption of tobacco discount coupons and other tobacco price promotions, and the policy has withstood legal challenges from the tobacco industry. ${ }^{20}$ Cessation programmes and healthcare providers counselling patients who are trying to quit smoking should warn them about these tobacco direct mail/email coupons and assist them to halt these mailings may increase their chances of successfully quitting smoking. ${ }^{21}$

Our analyses corroborate prior research that demonstrates disproportionate targeting of women and vulnerable populations by the tobacco industry's use of discount coupons. Female smokers, and individuals of lower SES (defined by education and poverty level in this study), were more likely than those of higher SES to report that they received tobacco direct mail/email coupons. This may represent active targeting of these populations by the tobacco industry, as revealed in their internal documents. ${ }^{13}$ This could increase or sustain smoking in these populations, as some work has found that women were more likely than men to use coupons in general, ${ }^{22}$ and low SES populations have a higher level of price sensitivity than higher SES populations. ${ }^{23}$ We also found that non-Hispanic black experimenters were more likely than their non-Hispanic white counterparts to have received these coupons. This is not surprising given that tobacco industry is known to target minority populations using various marketing strategies, price promotions included. ${ }^{24} 25$ The observations that non-Hispanic white never and current smokers were more likely than minority never and current smokers to have received these coupons is perhaps because the tobacco industry uses other marketing strategies to target these segments of the minority populations, for example, promoting menthol cigarettes and lowering sales prices in minority communities. ${ }^{24-26}$ Nonetheless, prohibiting tobacco direct mail/email coupons, and tobacco price promotions in general, may reduce SES and racial/ethnic disparities. A recent systematic review pointed out that while price promotion restrictions are one of the most common non-tax tobacco price policies, 


\section{What this paper adds}

- Previous studies suggested receipt of tobacco direct mail/ email coupons is associated with smoking behaviours.

- No studies to date have used a longitudinal design and a US nationally representative adult sample to examine how receiving tobacco coupons through direct mail/email influence smoking behaviours among adults at different stages of smoking behaviours.

- The current study found that receipt of tobacco direct mail/email coupons was disproportionately among lower socioeconomic adults. Receipts of these coupons also encourage and sustain smoking. Eliminating these coupons may reduce smoking in the population as well as its disparities.

how they impact average tobacco prices and smoking behaviours is largely unknown. ${ }^{27}$ Future studies are needed to evaluate or forecast the effects of these policies to facilitate their implementation.

The strengths of the PATH Study include the nationally representative adult sample, large sample size, detailed measurement of smoking behaviours and high retention rate to minimal selection bias due to attrition. Weighting the longitudinal sample also accounted for attrition to minimise the impact of selection bias on the associations assessed in this analysis. However, this study also has limitations. Receipt of tobacco direct mail/email coupons was based on self-report. It is possible that some participants may not correctly recall receiving these coupons in their mail/email. However, since participants in a previous study were able to reasonably recall the number of tobacco direct mail promotions received in the past 6 months, ${ }^{9}$ we believe the incorrect recall of exposure to tobacco direct mail/email coupons would have minimal impact on our findings.

In conclusion, in this first longitudinal study among a US nationally representative adult sample to assess the associations between exposure to tobacco direct mail/email coupons and trajectory of smoking behaviours, we found evidence that these coupons could promote the development of smoking behaviours, hinder smoking cessation, as well as facilitate smoking relapse. Fully implementing the tobacco price promotion ban in the USA as specified by the WHO MPOWER strategies could result in reduction in prevalence of smoking, as well as SES-related disparities in smoking. Future studies need to examine the impact of these policies on cigarette prices and smoking behaviours.

Contributors KC was responsible for study concept and drafted the manuscript. JCC conducted statistical analysis. All authors provided critical revision of the manuscript for important intellectual content. All authors approved the final version for publication.

Funding National Institute on Minority Health and Health Disparities Division of Intramural Research, and National Institute on Drug Abuse and Food and Drug Administration Center for Tobacco Products (K01DA037903).

Disclaimer Opinions and comments expressed in this article are authors' own and do not necessarily represent those of the US Government, Department of Health and Human Services, National Institutes of Health and National Institute on Minority Health and Health Disparities.

Competing interests None declared.

Patient consent Not required.

Ethics approval This research is a secondary data analysis of deidentified data and was determined by the National Institutes of Health Office of Health Subjects Research Protection to be exempted from review by an Institutional Review Board.

Provenance and peer review Not commissioned; externally peer reviewed.
Data sharing statement The data are available to the public.

(C) Article author(s) (or their employer(s) unless otherwise stated in the text of the article) 2019. All rights reserved. No commercial use is permitted unless otherwise expressly granted.

\section{REFERENCES}

1 Drope J, Schluger N, Cahn Z, et al. The tobacco atlas. Atlanta, GA: American Cancer Society and Vital Strategies, 2018.

2 National Cancer Institute. The role of the media in promoting and reducing tobacco use. Tobacco control monograph no. 19. Bethesda, MD: U.S. Department of Health and Human Services, National Institutes of Health, National Cancer Institute, 2008.

3 U.S. Department of Health and Human Services. The health consequences of smoking - 50 years of progress. A report of the surgeon general. Atlanta, GA: U.S. Department of Health and Human Services, Centers for Disease Control and Prevention, National Center for Chronic Disease Prevention and Health Promotion, Office on Smoking and Health, 2014.

4 Federal Trade Commission. Smokeless tobacco report for 2015. Washington, DC: Federal Trade Commission, 2017.

5 Federal Trade Commission. Cigarette report for 2015. Washington, DC: Federal Trade Commission, 2017.

6 Brock B, Schillo BA, Moilanen M. Tobacco industry marketing: an analysis of direct mail coupons and giveaways. Tob Control 2015;24:505-8.

7 Choi K, Forster JL. Frequency and characteristics associated with exposure to tobacco direct mail marketing and its prospective effect on smoking behaviors among young adults from the US Midwest. Am J Public Health 2014;104:2179-83.

8 Choi K, Soneji S, Tan ASL. Receipt of tobacco direct mail coupons and changes in smoking status in a nationally representative sample of US adults. Nicotine \& Tobacco Research 2017:94.

9 Choi K, Taylor N, Forster J. Sources and number of coupons for cigarettes and snus received by a cohort of young adults. Am J Health Promot 2018;32:153-60.

10 Nicksic NE, Snell LM, Rudy AK, et al. Tobacco marketing, e-cigarette susceptibility, and perceptions among adults. Am J Health Behav 2017;41:579-90.

11 World Health Organization. WHO report on the global tobacco epidemic, 2013 enforcing bans on tobacco advertising, promotion and sponsorship. Geneva, Switzerland: World Health Organization, 2013.

12 Tobacco Control Legal Consortium. Policy approaches to restricting tobacco product coupons and retail value-added promotions. Saint Paul, MN: Public Health Law Center 2013.

13 Brown-Johnson CG, England LJ, Glantz SA, et al. Tobacco industry marketing to low socioeconomic status women in the U.S.A. Tob Control 2014;23:e139-46.

14 Choi K, Hennrikus DJ, Forster JL, et al. Receipt and redemption of cigarette coupons, perceptions of cigarette companies and smoking cessation. Tob Control 2013:22:418-22

15 Mayhew KP, Flay BR, Mott JA. Stages in the development of adolescent smoking. Drug Alcohol Depend 2000;59(Suppl 1):61-81.

16 Hyland A, Ambrose BK, Conway KP, et al. Design and methods of the population assessment of tobacco and health (PATH) Study. Tob Control 2017;26:371-8.

17 U.S. Food and Drug Administration. Population assessment of tobacco and health (PATH) study [United States] restricted-use files, user guide. ICPSR36231-v13. Ann Arbor, MI: Inter-university Consortium for Political and Social Research, 2017.

18 World Health Organization. WHO report on the global tobacco epidemic, 2008: the MPOWER package. Geneva, Switzerland: World Health Organization, 2008.

19 El-Toukhy S, Choi K, Hitchman SC, et al. Banning tobacco price promotions, smokingrelated beliefs and behaviour: findings from the International Tobacco Control Four Country (ITC 4C) Survey. Tob Control 2018;27:310-8.

20 Liss S. Federal court upholds New York City's law to prohibit tobacco discounts and further reduce youth smoking. Washington, DC: Campaign Tob, Free Kids, 2014.

21 Lewis MJ, Delnevo CD, Slade J. Tobacco industry direct mail marketing and participation by New Jersey adults. Am J Public Health 2004;94:257-9.

22 Harmon SK, Jeanne Hill C. Gender and coupon use. Journal of Product \& Brand Management 2003;12:166-79.

23 Centers for Disease Control and Prevention (CDC). Response to increases in cigarette prices by race/ethnicity, income, and age groups--United States, 1976-1993. MMWR Morb Mortal Wkly Rep 1998;47:605-9.

24 White VM, White MM, Freeman K, et al. Cigarette promotional offers: who takes advantage? Am J Prev Med 2006;30:225-31.

25 Cruz TB, Wright LT, Crawford G. The menthol marketing mix: targeted promotions for focus communities in the United States. Nicotine Tob Res 2010;12(Suppl 2):S147-53.

26 Yerger VB, Przewoznik J, Malone RE. Racialized geography, corporate activity, and health disparities: tobacco industry targeting of inner cities. J Health Care Poor Underserved 2007;18(4 Suppl):10-38.

27 Golden SD, Smith MH, Feighery EC, et al. Beyond excise taxes: a systematic review of literature on non-tax policy approaches to raising tobacco product prices. Tob Control 2016:25:377-85. 